\title{
Preoperative Multi-Detector Computed Tomography for Isolated Surgical Aortic Valve Replacement; Planning for Future Transcatheter Options
}

\author{
Craig Basman ${ }^{1}$, Karthik Seetharam ${ }^{1}$, Joel Johnson ${ }^{2}$, Jonathan Hemli ${ }^{2}$, Derek Brinster ${ }^{1}$, \\ Nirav Patel ${ }^{1}$, S Scheinerman ${ }^{1}$, Chad Kliger ${ }^{1}$, and Luigi Pirelli ${ }^{1}$ \\ ${ }^{1}$ Lenox Hill Hospital \\ ${ }^{2}$ Affiliation not available
}

June 8, 2020

\begin{abstract}
ABSTRACT: Background: Valve-in-valve (ViV) transcatheter aortic valve replacement (TAVR) has become a valuable option in patients with bioprosthetic failure. However, potential issues with ViV TAVR may occur in patients with high risk anatomy for coronary obstruction and patients with baseline smaller bioprosthetic valves at risk for patient prosthesis mismatch. The purpose of this study was therefore to use preoperative electrocardiography (ECG)-gated, multidetector computed tomography (MDCT) in patients undergoing isolated surgical aortic valve replacement (SAVR) to 1) identify which would be high risk for coronary occlusion with ViV TAVR, and 2) predict intraoperative SAVR sizing. Methods: Among 223 patients from our institutions' database that underwent SAVR for aortic insufficiency (AI) or aortic stenosis (AS) between January 2012 and January 2020, 48 patients had MDCT imaging prior to surgery (AI; n=31, AS; $n=17$ ). Of all patients, 67\% ( $\mathrm{n}=32$ ) were bicuspid morphology. Results: With the use of virtual valve implantation, all patients with AI and bicuspid AS had feasible anatomy for ViV TAVR, while $38 \%$ of patients with tricuspid AS were high risk for coronary obstruction. There was a strong correlation between actual valve size implanted and preoperative MDCT measurements using annulus average diameter, area and/or perimeter. Conclusion: Preoperative MDCT in patients undergoing SAVR is a useful tool for lifetime management, particularly in patients with tricuspid AS. Decisions for surgical management may change based on MDCT's ability to predict intraoperative SAVR size and determine which patients may be high risk candidates for future ViV TAVR due to coronary artery obstruction.
\end{abstract}

Abbreviations:

AI- aortic insufficiency

AS- aortic stenosis

BASILICA- Bioprosthetic or native Aortic Scallop Intentional Laceration to prevent Coronary Artery obstruction

CABG- coronary artery bypass grafting

ECG- electrocardiography

MDCT- multi-detector computed tomography

PPM- patient prosthesis mismatch 
SAVR- surgical aortic valve replacement

TAVR- transcatheter aortic valve replacement

ViV- valve-in-valve

VTC- virtual ring to coronary ostial distance

\section{Introduction:}

Though the use of electrocardiography (ECG)-gated, multidetector computed tomography (MDCT) imaging is standard to determine if a patient with bioprosthetic aortic valve failure is a candidate for valve-in-valve $(\mathrm{ViV})$ transcatheter aortic valve replacement (TAVR), there is no recommendation for the use of preoperative MDCT for patients undergoing surgical aortic valve replacement (SAVR). Patients with bioprosthetic valve failure are frequently at high surgical risk for reoperation, which is why ViV TAVR has become a valuable option in such patients. (1) Due to the risk of coronary obstruction, there is a subset of patients not amenable to standard ViV TAVR without the use of advanced adjunctive techniques, such as chimney stenting or leaflet laceration (which both pose additional risks). $(2,3)$ Additionally, patients with smaller surgical prostheses may be predisposed to higher gradients post-ViV TAVR, which is associated with worsened quality of life and higher mortality rates. $(4,5)$ As further emphasis is placed on lifetime management of these patients, it is therefore important that we identify high-risk patients for successive procedures prior to initial surgical intervention. The purpose of this study was therefore 1) identify which patients undergoing SAVR would be high risk for ViV TAVR coronary occlusion based on MDCT, 2) use annular measurements on MDCT to predict intraoperative SAVR sizing.

\section{Methods:}

All patients who underwent isolated SAVR with a tissue valve between January 2012 and January 2020 were included in this study $(\mathrm{n}=154)$. Patients were excluded if they had prior cardiac surgery before the SAVR or did not have adequate MDCT images for analysis. (Figure 1) Clinical information was collected from the patient chart and surgical reports. MDCT images were analyzed by two experienced readers. Each reader was randomly assigned half of the scans to interpret. CT readers were blinded to patient data/operative reports.

\section{Surgical Aortic Valve Replacement}

All operations were performed using cardiopulmonary bypass with mild systemic hypothermia $\left(30-34{ }^{\circ} \mathrm{C}\right)$. The majority of procedures were performed with a mini-sternotomy or right thoracotomy, though there were $20 \%$ with full median sternotomy. Intraoperative assessment of the aortic annulus size was conducted after resection of the aortic valve cusps and complete debridement of the calcifications. The sizing obturator was then inserted into the annulus and the largest size valve possible was chosen. The replica end of the sizer was then used to ensure correct valve size. No patients underwent aortic root surgery, coronary artery bypass grafting (CABG) or coronary re-implantation (isolated SAVR only). Valve type was left up to the discretion of the operator. The majority of valves implanted were Edwards Magna Ease.

Annulus/Sinus/Root Measurements

To detect the annulus we obtained two measurements during an end-systolic phase. The annulus was defined as the virtual basal plane of attachment for the aortic valve leaflets. Measurements of the sinus of Valsalva (SOV), sinotubular junction (STJ) and coronary heights were obtained during diastolic phases.

Valve Sizing for MDCT measurements and Assessment for Future ViV Feasibility

We assumed that the sizer (which corresponds to the outer diameter of the valve frame) cannot be forced into an annulus smaller than the valve sizer (i.e.- an average diameter of 24.5 would not be able to accommodate a $25 \mathrm{~mm}$ valve, so it would be sized for a $23 \mathrm{~mm}$ valve). Based on the MDCT, we used the average annulus diameter to predict surgical valve sizing as follows; 
Average diameter of 19.0 to $20.9 \mathrm{~mm}=$ Valve size $19 \mathrm{~mm}$

Average diameter of 21 to $22.9 \mathrm{~mm}=$ Valve size $21 \mathrm{~mm}$

Average diameter of 23 to $24.9=$ Valve size $23 \mathrm{~mm}$

Average diameter of 25 to $26.9=$ Valve size $25 \mathrm{~mm}$

Average diameter of 27 to $28.9=$ Valve size $27 \mathrm{~mm}$

Averaged diameter $>29 \mathrm{~mm}=$ Valve size $29 \mathrm{~mm}$.

We then implanted a virtual valve with the same dimensions as a Carpentier-Edwards Magna Ease at the level of the annulus. We used this valve since it is the most common valve used at our institution. Subsequently, we were able to calculate a virtual valve to coronary ostial distance (VTC) and determine if the patient would be at risk for sinus sequestration, sealing of the aortic root with the virtual valve. We considered patients high risk for future ViV if VTC was $<4 \mathrm{~mm}$ and/or the virtual struts contacted the STJ without room for blood flow around. (6) (FIGURE 2, FIGURE 3)

MDCT Measurements versus Intraoperative Measurements

An important part of virtual valve placement is ensuring that we are placing a proper sized surgical valve. If our measurements on MDCT cannot predict actual valve implanted, then virtual valve sizing may be misguiding. Furthermore, the ability to properly predict SAVR size may be important for procedural planning (i.e.- in patients with small annuli, they might require root enlargement to reduce the risk of patient prosthesis mismatch (PPM)). We therefore evaluated how often we predicted the correct SAVR size implanted. We also assessed the correlation strength between annular diameter, area and perimeter compared to actual valve size implanted.

\section{Statistical Analysis}

For patient characteristics, continuous variables were reported as mean with standard deviation (SD) and compared between two groups using a 2-sample independent $t$ tests or Mann-Whitney U test (non-uniform data). A Pearson coefficient of determination $\left(\mathrm{R}^{2}\right)$ for all diameters, area and perimeter acquired by MDCT imaging was used for comparison with the intraoperative valve chosen. $\mathrm{R}^{2}$ values were considered very weak if $<0.3$, weak if $0.3-0.5$, moderate if $0.5-0.7$, strong if $>0.7$. (Moore D.S et al) $\mathrm{P}$ values [?]0.05 were considered statistically significant. All statistical analyses were performed with the use of SAS software, version 9.2 (SAS institute, Cary, NC).

\section{Results:}

Table 1 displays baseline patient characteristics. Of the 48 patients, $64 \%(\mathrm{n}=31)$ presented with AI and $36 \%(\mathrm{n}=17)$ presented with AS. Patients with AI were younger (59yrs vs 68.7yrs, $\mathrm{P}<0.01$ ) than patients with AS. Prevalence of bicuspid valves was $74 \%(\mathrm{n}=23)$ in patients with AI and $53 \%(\mathrm{n}=9)$ in patients with AS $(\mathrm{P}=0.11)$. Patients with AI had a larger annulus (annulus area; $554.77 \mathrm{~mm}^{2}+-123.2$ vs $474.18 \mathrm{~mm}^{2}+-$ $135.41, \mathrm{P}=0.049$ ) and STJ size (average STJ diameter; $31.49 \mathrm{~mm}+-3.67$ vs $28.49 \mathrm{~mm}+-3.28, \mathrm{P}=<0.01$ ) than those with AS. Of the 31 patients with bicuspid morphology, 9 (29\%) were Sieverts Type 0 and the rest were Sieverts Type $1(71 \%)$ based on MDCT evaluation.

The predominant valve used was Carpentier Edwards Magna Ease (75\%) while a Medtronic Mosaic valve was used $15 \%$ and Edwards Perimount 10\%. Among all AI patients, preoperative measurements based on MDCT predicted that no patients would be at high risk for coronary obstruction with ViV TAVR. Among patients with AS, the majority of patients (82\%) were not at high risk for ViV TAVR. Among the 18\% of patients $(\mathrm{n}=3)$ with high risk anatomy, the mechanism was sinus sequestration in one patient and leaflet obstruction (VTC $<4 \mathrm{~mm}$ ) in two patients. All patients with high risk anatomy had tricuspid AS. Of the tricuspid AS patients, $38 \%$ ( $\mathrm{n}=3$ ) were high risk for ViV TAVR (very low numbers - only 8 pts - limitation). In contrary, there were no high risk patients with bicuspid AS. (FIGURE 4) 
In patients with AI, MDCT properly predicted valve size in $71 \%$ of patients. The other $29 \%$ had a smaller valve implanted (MDCT overestimated the measurements). In patients with AS, MDCT properly predicted valve size in $94 \%$ of patients (all but one patient). There was only one patient in which MDCT underestimated the measurement (a patient with tricuspid AS, in which the annular average diameter was $23.5 \mathrm{~mm}$, and a $25 \mathrm{~mm}$ valve was implanted). Table 2 displays how often the valve size was correctly predicted based on MDCT measurements. In patients with AI there was strong correlation between actual valve size implanted and annulus average diameter $\left(\mathrm{R}^{2}=0.72, \mathrm{P}<0.01\right)$, area $\left(\mathrm{R}^{2}=0.73, \mathrm{P}<0.01\right)$ and perimeter $\left(\mathrm{R}^{2}=0.70, \mathrm{P}<0.01\right)$ on MDCT. (FIGURE 5) In patients with AS, there was also a strong correlation between actual valve size implanted and annulus average diameter $\left(\mathrm{R}^{2}=0.90, \mathrm{P}<0.01\right)$, area $\left(\mathrm{R}^{2}=0.85, \mathrm{P}<0.01\right)$ and perimeter $\left(\mathrm{R}^{2}=0.90, \mathrm{P}<0.01\right) .($ FIGURE 6$)$

\section{Discussion:}

In our study, we found that in patients undergoing SAVR with preoperative MDCT imaging; (1) There was a strong correlation between annular measurements on MDCT and actual SAVR size implanted; 2) Using pre-procedural virtual valve implantation, all patients with AI were predicted to be low risk for ViV TAVR coronary obstruction; (3) In patients with AS, pre-procedural MDCT with virtual valve implantation found that $38 \%$ of patients with tricuspid AS would be at high risk, while no patients with bicuspid AS were at high risk.

To our knowledge, this is the first paper addressing the issue of whether a patient undergoing SAVR will be a candidate for ViV TAVR in the future based on pre-procedural MDCT. As our surgical and transcatheter techniques evolve, focus has shifted to lifetime management of valvular disease. Patients with bioprosthetic valve failure are frequently at high surgical risk for reoperation, which is why ViV TAVR has become a valuable option in such patients. (1) Coronary artery obstruction more commonly occurs with ViV TAVR, and is a potentially fatal complication that portends a mortality rate greater than 50\%. (6) Patients at high risk for coronary obstruction during ViV TAVR still have options, however these options come at a higher risk. Chimney stenting is associated with higher risk of procedural and delayed clinical events. (8) Which prompted the advent of BASILICA (Bioprosthetic or native Aortic Scallop Intentional Laceration to prevent Coronary Artery obstruction). However, there are a significant percentage of patients that are not candidates for BASILICA, and the procedure may result in higher rates of procedural complications including stroke. (3) Therefore, if we can classify patients who will be high risk for future transcatheter procedures, it may factor into the shared decision making between the patient and heart team. Options may then include; 1) TAVR as primary strategy followed by SAVR for failure, 2) SAVR with surgical manipulation (e.g.- coronary re-implant, root enlargement) to decrease subsequent risk for coronary obstruction, 3) Standard SAVR (with the awareness that advanced adjunctive techniques will likely be necessary or transcatheter options limited in the future).

Though the majority of patients (74\%) were correctly sized with MDCT, the rest of the patients (26\%) were oversized compared to actual SAVR implanted. One would expect that virtual implantation of a larger valve would increase the chance of falsely predicting high risk anatomy for ViV TAVR. Interestingly, despite implanting a larger virtual valve in almost $1 / 4$ of patients, MDCT did not predict any patients with AI to be at high risk for coronary obstruction. These findings suggest that outside of MDCT for root sizing/coronary evaluation, preoperative MDCT is less helpful for patients with AI.

MDCT predicted the correct valve size implanted in all but one patient with AS (94\%). There was a strong correlation between MDCT annular measurements and actual SAVR sizing. In patients with bicuspid AS, all patients that underwent virtual valve implantation were predicted to have low risk anatomy for ViV TAVR. Perhaps this is because root sizes are generally larger in bicuspid AS patients. With virtual valve implantation, $38 \%$ of patients with tricuspid AS were high risk for ViV TAVR. This warrants a bigger question; would preoperative MDCT change management in patients with tricuspid AS with plan for SAVR? Future studies are needed to 1) compare preoperative virtual valve implantation of the SAVR to postoperative MDCT and 2) determine if preoperative MDCT can safely change the lifetime management in tricuspid AS patients. 
As the heart team plans for the treatment of each individual's aortic valve pathology, it is important to determine what size surgical bioprosthetic valve can be implanted. Surgical prosthesis with small internal orifice diameters may predispose patients to transcatheter heart valve under-expansion at the time of ViV TAVR. Smaller sized surgical valves are associated with higher post-procedural gradients and increased mortality in patients undergoing ViV TAVR. $(4,5)$ The ability to predict SAVR sizing can therefore be useful to identify patients that require smaller valves. For example, if preoperative workup reveals that the annulus will only accommodate a $19 \mathrm{~mm}$ valve, the heart team can then discuss the most suitable option for the lifetime management of each case. Options may include; standard SAVR, SAVR with root enlargement, implantation of a prosthetic valve with an expandable frame (i.e.- Inspiris, Edwards Lifescience, Irvine), mechanical valve, or the use of TAVR instead of SAVR. This knowledge is especially useful in patients with AS, who tend to have smaller annuli than patients with AI (annulus area; $554.77 \mathrm{~mm}^{2}+-123.2$ vs $\left.474.18 \mathrm{~mm}^{2}+-135.41, \mathrm{P}=0.049\right)$.

Among our studies limitations was the lack of patients with preoperative MDCT. This is reflective of current standard practice, which does not necessitate preoperative MDCT. Larger numbers are needed to evaluate what percentage of AS patients would be high risk based on preoperative MDCT. We also concede that virtual valve implantation has never proven to predict feasibility for future ViV TAVR procedures. Though outer stent diameters may be the same between valve manufacturers, valve sizes may have different inner stent diameters, and therefore a patient may end up with a different sized valve depending on the manufacturer. If a smaller or larger prosthesis is implanted, it will skew measurements and virtual valve implantation may be less accurate. Additionally, valves may be canted one way or implanted at different depths, therefore studies will need to address a pre-SAVR virtual valve implantation with MDCT to post-SAVR measurements on MDCT. Unfortunately, it is not standard protocol to obtain post-MDCT, so we were not able to investigate this. However, the majority of valve implantations on pre-CT were correctly sized for actual intraoperative measurements (or within 1 size at least), so this should not factor in largely to the limitations.

We believe that our study addresses a pressing need to investigate the utility of MDCT to help guide management decisions for patients undergoing isolated SAVR. Though the surge of TAVR has led to a reduction in isolated SAVR volume, SAVR is still an option for low-risk patients. $(1,9)$ It is the low risk/younger patients that are more likely to need a valve re-intervention during their lifetime. Hence, shared decision making between the patient and heart team is essential in patients with high risk anatomy to discuss all options for lifetime management.

\section{Conclusion:}

Preoperative MDCT in patients undergoing SAVR is a useful tool for lifetime management, particularly in patients with tricuspid AS. Decisions for surgical management may change based on MDCT's ability to predict intraoperative SAVR size and determine which patients may be high risk candidates for future ViV TAVR due to coronary artery obstruction.

\section{References:}

1. Nishimura RA., Otto CM., Bonow RO, Carabello BA, Erwin JP, Fleisher LA, et al. 2017 AHA/ACC focused update of the 2014 AHA/ACC guideline for the management of patients with valvular heart disease: a report of the American College of Cardiology/American Heart Association Task Force on clinical practice guidelines. Circulation 2017; 135: e1159-e1195.doi:10.1161/CIR.0000000000000503

2. Abramowitz Y, Chakravarty T, Jilaihawi H, Kashif M, Kazuno Y, Takahashi N, et al. Clinical impact of coronary protection during transcatheter aortic valve implantation: first reported series of patients. EuroIntervention 2015; 11: 572-581.doi:10.4244/EIJV11I5A112

3. Khan JM., Dvir D., Greenbaum AB, Babaliaros VC, Rogers T, Aldea G et al. Transcatheter laceration of aortic leaflets to prevent coronary obstruction during transcatheter aortic valve replacement: concept to first-in-human. JACC Cardiovasc Interv 2018; 11: 677-689.doi:10.1016/j.jcin.2018.01.247

4. Bapat V.N, Attia R, Thomas M. Effect of valve design on the stent internal diameter of a bioprosthetic valve: a concept of true internal diameter and its implications for the valve-in-valve procedure. J Am 
Coll Cardiol Intv, 7 (2014), pp. 115-127

5. Dvir D, Webb JG1, Bleiziffer S, Pasic M, Waksman R, Kodali S, Barbanti M, et al. Valve-in-Valve International Data Registry Investigators. Transcatheter aortic valve implantation in failed bioprosthetic surgical valves. JAMA. 2014 Jul;312(2):162-70. doi: 10.1001/jama.2014.7246.

6. Ribeiro H., Rodes-Cabau J, Blanke P, Leipsic J, Park JK, Bapat V, et al. Incidence, predictors, and clinical outcomes of coronary obstruction following transcatheter aortic valve replacement for degenerative bioprosthetic surgical valves: insights from the VIVID registry. Eur Heart J 2018; 39: 687-69

7. Moore DS, Notz WI, Flinger MA. The basic practice of statistics (6th ed.). 2013. New York, NY: W.H. Freeman and Company. Page 138

8. Mercanti F, Rosseel L, Neylon A, Bagur R, Sinning JM, Nickenig G, et al. Chimney Stenting for Coronary Occlusion During TAVR. J Am Coll Cardiol Intv. 2020 Mar, 13 (6) 751-761.

9. Kundi H, Strom JB, Valsdottir LR, Elmariah S, Poplma JJ, Shen C et al. Trends in Isolated Surgical Aortic Valve Replacement According to Hospital-Based Transcatheter Aortic Valve Replacement Volume. J Am Coll Cardiol Intv. 2018 Nov, 11 (21) 2148-2156.

TABLES:

Table 1 (BASELINE/ANATOMICAL/PROCEDURAL CHARACTERISTICS)

\begin{tabular}{|c|c|c|c|c|}
\hline & AI & & AS & \\
\hline $\mathrm{n}$ & 31 & & 17 & \\
\hline Age, years & $59.16 \pm 14.87$ & $59.16 \pm 14.87$ & $68.71 \pm 8.68$ & $68.71 \pm$ \\
\hline BMI & $31.1 \pm 11.75$ & $31.1 \pm 11.75$ & $28.31 \pm 6.62$ & $28.31 \pm$ \\
\hline Female Sex, n (\%) & $6(19 \%)$ & & $3(17 \%)$ & \\
\hline Creatinine, mg/dL & $1.16 \pm 0.97$ & $1.16 \pm 0.97$ & $0.92 \pm 0.22$ & $0.92 \pm$ \\
\hline Ejection Fraction (\%) & $55.4 \pm 11.59$ & $55.4 \pm 11.59$ & $57.71 \pm 12.97$ & $57.71 \pm$ \\
\hline CHF, n (\%) & $16(52 \%)$ & & $10(59 \%)$ & \\
\hline PVD, n (\%) & $7(22.5 \%)$ & & $3(17.6 \%)$ & \\
\hline MI, n (\%) & $3(9.7 \%)$ & & $0(0 \%)$ & \\
\hline COPD, n $(\%)$ & $1(3 \%)$ & & $1(6 \%)$ & \\
\hline Diabetes, n $(\%)$ & $4(13 \%)$ & & $8(47 \%)$ & \\
\hline Renal Dialysis, n (\%) & $2(6.4 \%)$ & & $0(0 \%)$ & \\
\hline ANATOMIC FINDINGS ON MDCT & ANATOMIC FINDINGS ON MDCT & & & \\
\hline Bicuspid Aortic Valve, n (\%) & $23(74 \%)$ & & $9(53 \%)$ & \\
\hline Average STJ size $(\mathrm{mm})$ & $31.49 \pm 3.67$ & $31.49 \pm 3.67$ & $28.49 \pm 3.28$ & $28.49 \pm$ \\
\hline RCA height (mm) & $17.84 \pm 4.43$ & $17.84 \pm 4.43$ & $14.5 \pm 5.58$ & $14.5 \pm$ \\
\hline LCA height (mm) & $17.36 \pm 4.7$ & $17.36 \pm 4.7$ & $16.74 \pm 3.3$ & $16.74 \pm$ \\
\hline Annulus area $\left(\mathrm{mm}^{2}\right)$ & $554.77 \pm 123.2$ & $554.77 \pm 123.2$ & $474.18 \pm 135.41$ & 474.18 \\
\hline Annulus Perimeter $\left(\mathrm{mm}^{2}\right)$ & $86.31 \pm 9.45$ & $86.31 \pm 9.45$ & $78.81 \pm 10.88$ & $78.81 \pm$ \\
\hline Average Diameter $\left(\mathrm{mm}^{2}\right)$ & $26.41 \pm 2.77$ & $26.41 \pm 2.77$ & $24.42 \pm 3.11$ & $24.42 \pm$ \\
\hline $\operatorname{MaxSOV}(\mathrm{mm})$ & $36.5 \pm 3.45$ & $36.5 \pm 3.45$ & $34.29 \pm 6.23$ & $34.29 \pm$ \\
\hline MinSOV (mm) & $32.74 \pm 4.30$ & $32.74 \pm 4.30$ & $31.26 \pm 4.36$ & $31.26 \pm$ \\
\hline
\end{tabular}

Legend: All values mean \pm SD. AI; Aortic Insufficiency, AS; Aortic Stenosis, BMI; body mass index; CHF; congestive heart failure, PVD; peripheral vascular, MI; previous myocardial infarction, COPD; chronic obstructive pulmonary disease, STJ; Sinotubular junction, RCA; right coronary artery height above annulus, LMCA; lef main coronary artery height, MaxSOV; maximum sinus of valsalva measurement, MinSOV; minimum sinus of valsalva measurement

Table 2: Predicted Measurements on MDCT vs Actual Surgical Valve Implanted 


\begin{tabular}{lllll}
\hline & Correct & Undersized & Oversized & \% Correct \\
\hline AI & 22 & 0 & 9 & $71 \%$ \\
AS & 16 & 1 & 0 & $94 \%$ \\
\hline
\end{tabular}

\section{FIGURE LEGENDS}

Figure 1. Flow-chart of patient selection.

Figure 2. MDCT analysis of a patient low risk for coronary obstruction with ViV

A: Based on an average annulus size measured at $21.3 \mathrm{~mm}$, a virtual $21 \mathrm{~mm}$ valve implanted

B: It is evident that the strut height will not reach the level of the STJ (Cross sectional view of STJ, with avg diameter of $32.6 \mathrm{~mm}$ )

C: At the level of the left coronary artery (LCA), the VTC measures $>5 \mathrm{~mm}$ (low risk for coronary obstruction)

Figure 3. MDCT analysis of a patient high risk for coronary obstruction with ViV

A: Based on an annulus size (Avg 21.9), a virtual $21 \mathrm{~mm}$ valve implanted. It is evident that the strut height will be above the low-rising RCA artery)

B: The virtual valve does not reach the level of the STJ, so there is no risk of sinus sequestration

C: VTC measures $2.08 \mathrm{~mm}$, which we would classify as high risk for coronary obstruction

Figure 4: ViV Feasibility

Figure 5: Correlation between MDCT measurements and actual valve size implanted in patients with aortic insufficiency

Figure 6: Correlation between MDCT measurements and actual valve size implanted in patients with aortic stenosis

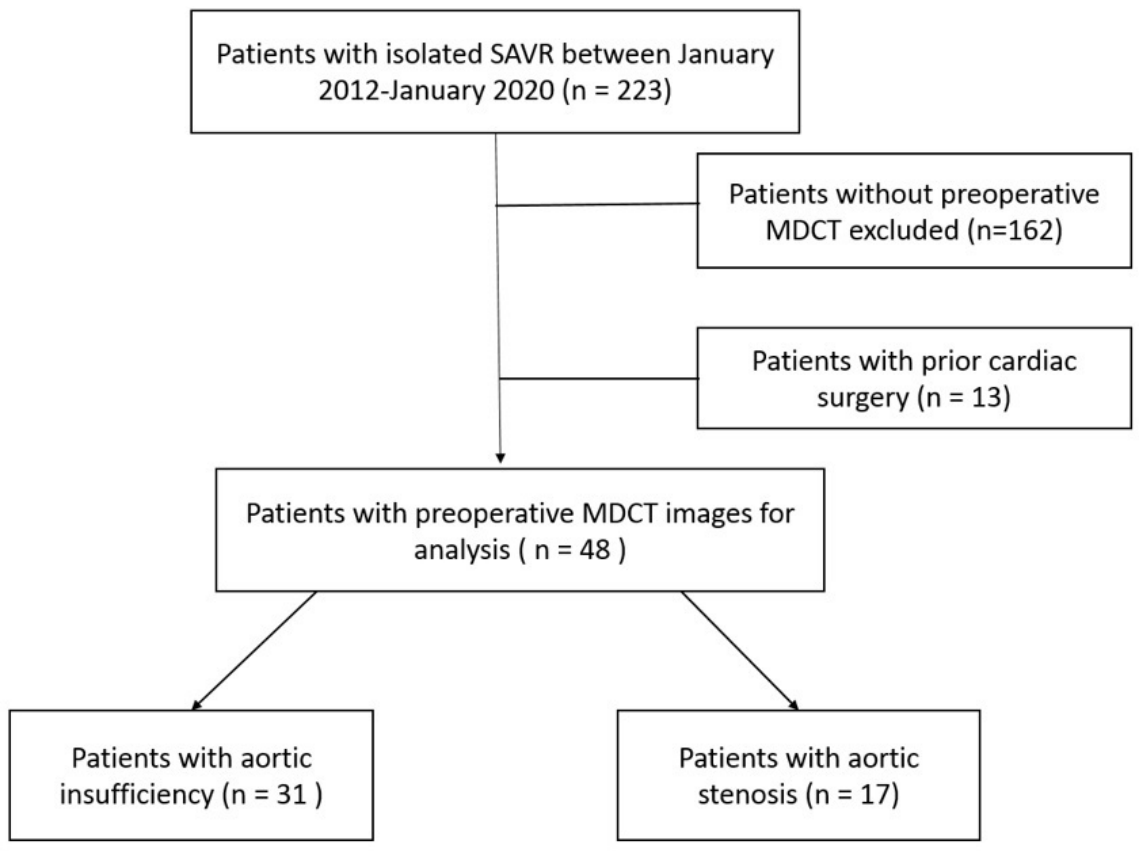



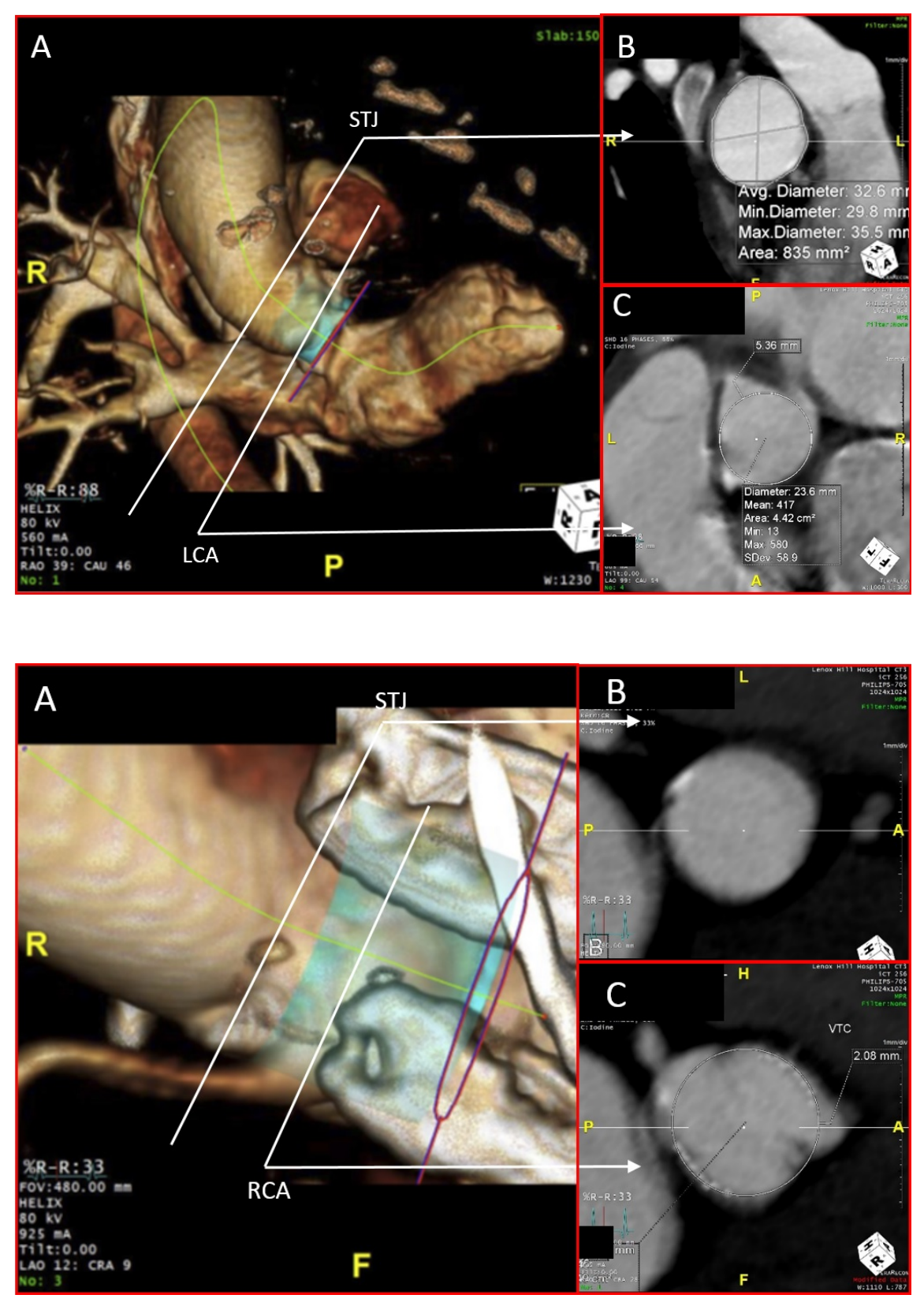

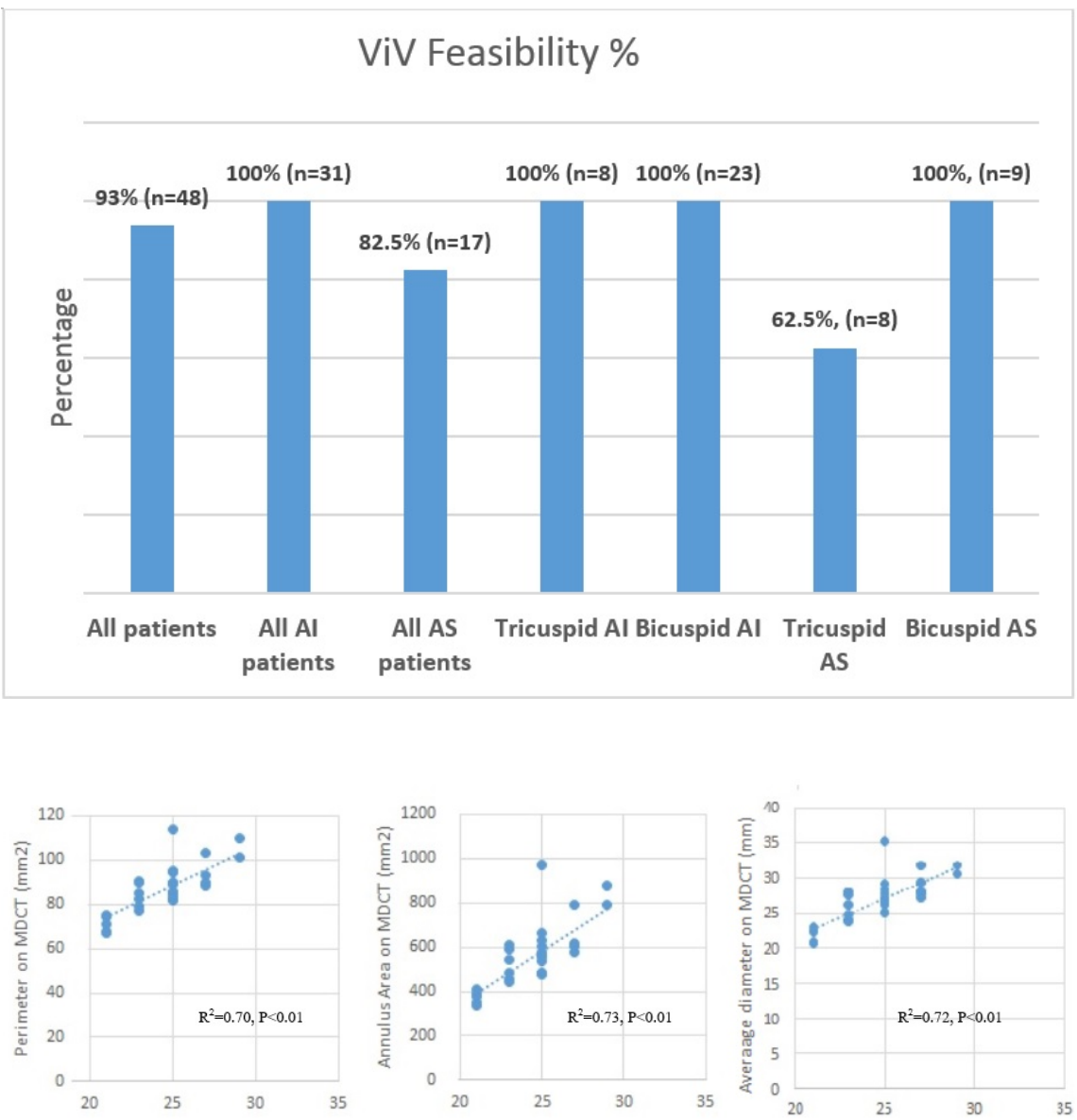

Valve Size Implanted (mm)
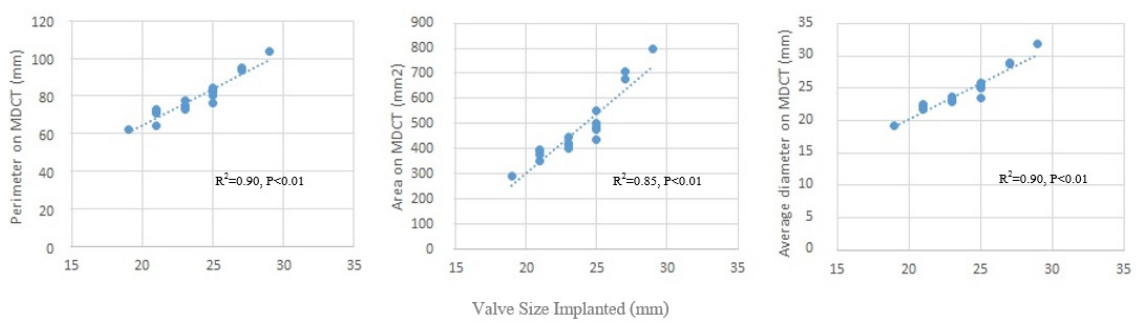